\title{
Cricoarytenoid Joint
}

National Cancer Institute

\section{Source}

National Cancer Institute. Cricoarytenoid Joint. NCI Thesaurus. Code C32397.

The synovial joints uniting the base of each arytenoid cartilage to the lamina of the cricoid cartilage. 